\title{
A Local Fractional Integral Inequality on Fractal Space Analogous to Anderson's Inequality
}

\author{
Wei Wei, ${ }^{1,2}$ H. M. Srivastava, ${ }^{3}$ Yunyi Zhang, ${ }^{4}$ Lei Wang, ${ }^{1}$ Peiyi Shen, ${ }^{5}$ and Jing Zhang ${ }^{1}$ \\ ${ }^{1}$ School of Computer Science and Engineering, Xi'an University of Technology, Xian 710048, China \\ ${ }^{2}$ Shaanxi Key Laboratory for Network Computing and Security Technology, Xian University of Technology, Xian 710048, China \\ ${ }^{3}$ Department of Mathematics and Statistics, University of Victoria, Victoria, BC, Canada V8W 3R4 \\ ${ }^{4}$ College of Computer and Communication Engineering, Zhengzhou University of Light Industry, Dongfeng Road, \\ Zhengzhou, Henan Province, China \\ ${ }^{5}$ National School of Software, Xidian University, Xian 710071, China
}

Correspondence should be addressed to Wei Wei; weiwei@xaut.edu.cn

Received 8 April 2014; Accepted 18 May 2014; Published 2 June 2014

Academic Editor: Xiao-Jun Yang

Copyright (C) 2014 Wei Wei et al. This is an open access article distributed under the Creative Commons Attribution License, which permits unrestricted use, distribution, and reproduction in any medium, provided the original work is properly cited.

Anderson's inequality (Anderson, 1958) as well as its improved version given by Fink (2003) is known to provide interesting examples of integral inequalities. In this paper, we establish local fractional integral analogue of Anderson's inequality on fractal space under some suitable conditions. Moreover, we also show that the local fractional integral inequality on fractal space, which we have proved in this paper, is a new generalization of the classical Anderson's inequality.

\section{Introduction}

In the year 1958, Anderson [1] established the following very interesting result.

Theorem 1. If $F_{i}(x)$ is convex increasing on $[0,1]$ and $F_{i}(0)=0$ for each $i=1,2, \ldots, n$, then

$$
\begin{aligned}
& \int_{0}^{1} F_{1}(x) F_{2}(x) \cdots F_{n}(x) \mathrm{d} x \\
& \quad \geqq \frac{2 n}{n+1}\left(\int_{0}^{1} F_{1}(x) \mathrm{d} x\right) \cdots\left(\int_{0}^{1} F_{n}(x) \mathrm{d} x\right) .
\end{aligned}
$$

Subsequently, Fink [2] improved Anderson's inequality (1) to the following form.
Theorem 2. If $F_{i}(x) / x$ is increasing on $(0,1]$ and $F_{i}(0)=0$ for each $i=1,2, \ldots, n$, then

$$
\begin{aligned}
& \int_{0}^{1} F_{1}(x) F_{2}(x) \cdots F_{n}(x) \mathrm{d} x \\
& \quad \geqq \frac{2^{n}}{n+1}\left(\int_{0}^{1} F_{1}(x) \mathrm{d} x\right) \cdots\left(\int_{0}^{1} F_{n}(x) \mathrm{d} x\right) .
\end{aligned}
$$

Moreover, Fink [2] also pointed out that the condition $F_{i}(0)=0(i=1,2, \ldots, n)$ in Theorems 1 and 2 cannot be dropped.

In recent years, the local fractional calculus has received significantly remarkable attention from scientists and engineers. Some of the concepts of the local fractional derivative were established in [3-26]. In particular, the local fractional derivative was introduced in [3-9, 17, 21-26], Jumarie modified the Riemann-Liouville derivative in $[10,11]$, and the fractal derivative was proposed in $[12-16,18-20]$. As a result, the theory of local fractional calculus plays an important 
role in applications in several different fields such as theoretical physics $[5,9]$, the theory of elasticity and fracture mechanics [5], and so on. For example, in [9], the authors proposed the local fractional Fokker-Planck equation. The local fractional Stieltjes transform was established in [27]. The fractal heat conduction problems were presented in [5, 18]. Local fractional improper integral was obtained in [28]. The principles of virtual work and minimum potential and complementary energy in the mechanics of fractal media were investigated in [5]. Local fractional continuous wavelet transform was studied in [29]. Mean-value theorems for local fractional integrals were considered in [30]. In [31], the authors dealt with fractal wave equations. The finite YangLaplace transform was introduced in [32]. Local fractional Schrödinger equation was studied in [33]. The local fractional Hilbert transform was given in [34]. The wave equation on Cantor sets was considered in [35]. The diffusion problems in fractal media were reported in [15] (see also several other recent developments on fractional calculus and local fractional calculus presented in [36-41]).

The purpose of this paper is to establish a certain local fractional integral inequality on fractal space, which is analogous to Anderson's inequality asserted by Theorem 1 . This paper is divided into the following three sections. In Section 2, we recall some basic facts about local fractional calculus. In Section 3, the main result is presented.

\section{Preliminaries}

In this section, we would review the basic notions of local fractional calculus (see [3-5]).

2.1. Local Fractional Continuity of Functions. In order to study the local fractional continuity of nondifferentiable functions on fractal sets, we first give the following results on the local fractional continuity of functions.

Lemma 3 (see [5]). Suppose that $\mathbb{F}$ is a subset of the real line and is a fractal. Suppose also that $f:(\mathbb{F}, d) \rightarrow\left(\Omega^{\prime}, d^{\prime}\right)$ is a bi-Lipschitz mapping. Then there are two positive constants $\rho$ and $\tau$, and $\mathbb{F} \subset \mathbb{R}$ :

$$
\rho^{s} H^{s}(\mathbb{F}) \leqq H^{s}(f(\mathbb{F})) \leqq \tau^{s} H^{s}(\mathbb{F}) \quad(\mathbb{F} \subset \mathbb{R}),
$$

such that, for all $x_{1}, x_{2} \in \mathbb{F}$,

$$
\rho^{\alpha}\left|x_{1}-x_{2}\right|^{\alpha} \leqq\left|f\left(x_{1}\right)-f\left(x_{2}\right)\right| \leqq \tau^{\alpha}\left|x_{1}-x_{2}\right|^{\alpha} \text {. }
$$

From Lemma 3, it is easily seen that (see [5])

$$
\left|f\left(x_{1}\right)-f\left(x_{2}\right)\right| \leqq \tau^{\alpha}\left|x_{1}-x_{2}\right|^{\alpha} \quad\left(x_{1}, x_{2} \in \mathbb{F}\right)
$$

so that

$$
\left|f\left(x_{1}\right)-f\left(x_{2}\right)\right| \leqq \varepsilon^{\alpha} \quad\left(x_{1}, x_{2} \in \mathbb{F}\right),
$$

where $\alpha$ is the fractal dimension of $\mathbb{F}$.
Definition 4 (see $[3,5])$. Assume that there exists

$$
\left|f(x)-f\left(x_{0}\right)\right| \leqq \varepsilon^{\alpha},
$$

with

$$
\left|x-x_{0}\right|^{\alpha} \leqq \delta^{\alpha}
$$

for $\varepsilon, \delta>0$ and $\varepsilon, \delta \in \mathbb{R}$. Then $f(x)$ is said to be local fractional continuous at $x=x_{0}$, denoted by

$$
\lim _{x \rightarrow x_{0}} f(x)=f\left(x_{0}\right) .
$$

The function $f(x)$ is local fractional continuous on the interval $(a, b)$, denoted by (see [5])

$$
f(x) \in C_{\alpha}(a, b)
$$

if (7) holds true for $x \in(a, b)$.

Definition 5 (see $[4,5]$ ). Assume that $f(x)$ is a nondifferentiable function of exponent $\alpha(0<\alpha \leqq 1)$. Then $f(x)$ is called the Hölder function of exponent $\alpha$ if, for $x, y \in \mathbb{F}$, one has

$$
|f(x)-f(y)| \leqq C|x-y|^{\alpha} \quad(0<\alpha \leqq 1) .
$$

Definition 6 (see $[4,5]$ ). A function $f(x)$ is said to be continuous of order $\alpha(0<\alpha \leqq 1)$ or, equivalently, $\alpha$ continuous, if

$$
\left|f(x)-f\left(x_{0}\right)\right| \leqq o\left(\left(x-x_{0}\right)^{\alpha}\right) \quad(0<\alpha \leqq 1) .
$$

\subsection{Local Fractional Derivatives and Local Fractional Integrals}

Definition 7 (see [3-5]). Assume that $f(x) \in C_{\alpha}(a, b)$. Then a local fractional derivative of $f(x)$ of order $\alpha$ at $x=x_{0}$ is defined by

$$
f^{(\alpha)}\left(x_{0}\right)=\left.\frac{d^{\alpha} f(x)}{d x^{\alpha}}\right|_{x=x_{0}}=\lim _{x \rightarrow x_{0}} \frac{\Delta^{\alpha}\left(f(x)-f\left(x_{0}\right)\right)}{\left(x-x_{0}\right)^{\alpha}},
$$

where

$$
\Delta^{\alpha}\left(f(x)-f\left(x_{0}\right)\right) \cong \Gamma(1+\alpha) \Delta\left(f(x)-f\left(x_{0}\right)\right) .
$$

It follows from Definition 7 that there exists (see [5])

$$
f(x) \in D_{x}^{(\alpha)}(a, b)
$$

if

$$
f^{(\alpha)}(x)=D_{x}^{(\alpha)} f(x)
$$

for any $x \in(a, b)$.

Definition 8 (see $[3,5])$. (a) If $f^{(\alpha)}(x)>0$ on a given interval, then $f(x)$ is increasing on that interval.

(b) If $f^{(\alpha)}(x)<0$ on a given interval, then $f(x)$ is decreasing on that interval. 
Definition 9 (see [42]). A function $f(x)$ is called $\alpha$-convex on $I$ if the following inequality holds true:

$$
f\left(\lambda x_{1}+(1-\lambda) x_{2}\right) \leqq \lambda^{\alpha} f\left(x_{1}\right)+(1-\lambda)^{\alpha} f\left(x_{2}\right)
$$

for all $x_{1}, x_{2} \in I$ and $0 \leqq \lambda \leqq 1$ such that $\lambda x_{1}+(1-\lambda) x_{2} \in I$.

Theorem 10 (see [42]). Assume that $f(x)$ is an $\alpha$-local differentiable function on I. If $f^{(\alpha)}(x)$ is nondecreasing (nonincreasing) on $I$, then the function $f$ is $\alpha$-convex ( $\alpha$-concave) on $I$.

Theorem 11 (see [42]). Assume that $f(x)$ is a local fractional continuous function. Then each of the following assertions holds true:

(1) if $f^{(2 \alpha)}(x)$ exists on I and $f^{(2 \alpha)}(x) \geqq 0$ for all $x \in I$, then $f$ is $\alpha$-convex on $I$;

(2) if $f^{(2 \alpha)}(x)$ exists on I and $f^{(2 \alpha)}(x) \leqq 0$ for all $x \in I$, then $f$ is $\alpha$-concave on $I$.

Definition 12 (see [3-5]). Assume that $f(x) \in C_{\alpha}(a, b)$. A local fractional integral of $f(x)$ of order $\alpha$ in the interval $[a, b]$ is expressed by

$$
\begin{aligned}
{ }_{a} I_{b}^{(\alpha)} f(x) & =\frac{1}{\Gamma(1+\alpha)} \int_{a}^{b} f(t)(\mathrm{d} t)^{\alpha} \\
& =\frac{1}{\Gamma(1+\alpha)} \lim _{\Delta t \rightarrow 0} \sum_{j=0}^{N-1} f\left(t_{j}\right)\left(\Delta t_{j}\right)^{\alpha},
\end{aligned}
$$

where

$$
\begin{gathered}
\Delta t_{j}=t_{j+1}-t_{j} \quad(j=0,1, N-1), \\
\Delta t=\max \left\{\Delta t_{1}, \Delta t_{2}, \ldots, \Delta t_{j}, \ldots\right\}, \\
{\left[t_{j}, t_{j+1}\right] \quad(j=0,1, \ldots, N-1) \quad\left(t_{0}=a ; t_{N}=b\right)}
\end{gathered}
$$

are a partition of the interval $[a, b]$.

It follows from Definition 12 that (see [5])

$$
f(x) \in{ }_{a} I_{x}^{(\alpha)}(a, b),
$$

if

$$
{ }_{a} I_{x}^{(\alpha)} f(x)
$$

for any $x \in(a, b)$.

Remark 13 (see [3-5]). Assume that $f(x) \in D_{x}^{(\alpha)}(a, b)$ or $f(x) \in C_{\alpha}(a, b)$; then

$$
f(x) \in{ }_{a} I_{x}^{(\alpha)}(a, b) .
$$

\section{Main Results}

Lemma 14. Let $f(x), g(x) \in C_{\alpha}(0,1)$ satisfy the constraints that $f(0)=0$ and $g(x)$ is increasing on $(0,1]$. If the function

$$
\frac{f(x)}{\Gamma(1+2 \alpha) x^{\alpha} / \Gamma(1+\alpha)}
$$

is increasing on $(0,1]$, then

$$
\begin{aligned}
& \frac{1}{\Gamma(1+\alpha)} \int_{0}^{1} f(x) g(x)(\mathrm{d} x)^{\alpha} \\
& \quad \geqq \frac{1}{\Gamma(1+\alpha)} \int_{0}^{1} f^{*}(x) g(x)(\mathrm{d} x)^{\alpha},
\end{aligned}
$$

where

$$
f^{*}(x)=\frac{\Gamma(1+2 \alpha) x^{\alpha}}{\Gamma(1+\alpha)} \cdot \frac{1}{\Gamma(1+\alpha)} \int_{0}^{1} f(u)(\mathrm{d} u)^{\alpha}
$$

for $x \in[0,1]$.

Proof. Let

$$
H(x)=\frac{1}{\Gamma(1+\alpha)} \int_{0}^{x}\left[f^{*}(t)-f(t)\right](\mathrm{d} t)^{\alpha} \quad(x \in[0,1]) .
$$

Then, clearly, $H(0)=0$ and

$$
\begin{aligned}
H(1) & =\frac{1}{\Gamma(1+\alpha)} \int_{0}^{1}\left[f^{*}(t)-f(t)\right](\mathrm{d} t)^{\alpha} \\
& =\frac{1}{\Gamma(1+\alpha)} \int_{0}^{1} f^{*}(t)(\mathrm{d} t)^{\alpha}
\end{aligned}
$$

$$
\begin{gathered}
-\frac{1}{\Gamma(1+\alpha)} \int_{0}^{1} f(t)(\mathrm{d} t)^{\alpha} \\
=\frac{1}{\Gamma(1+\alpha)} \int_{0}^{1} \frac{\Gamma(1+2 \alpha) t^{\alpha}}{\Gamma(1+\alpha)}
\end{gathered}
$$$$
\frac{1}{\Gamma(1+\alpha)} \int_{0}^{1} f(u)(\mathrm{d} u)^{\alpha}(\mathrm{d} t)^{\alpha}
$$$$
-\frac{1}{\Gamma(1+\alpha)} \int_{0}^{1} f(u)(\mathrm{d} u)^{\alpha}
$$$$
=\frac{1}{\Gamma(1+\alpha)} \int_{0}^{1} \frac{\Gamma(1+2 \alpha) t^{\alpha}}{\Gamma(1+\alpha)}(\mathrm{d} t)^{\alpha}
$$

$$
\begin{aligned}
& \times \frac{1}{\Gamma(1+\alpha)} \int_{0}^{1} f(u)(\mathrm{d} u)^{\alpha} \\
& -\frac{1}{\Gamma(1+\alpha)} \int_{0}^{1} f(u)(\mathrm{d} u)^{\alpha} \\
= & \left(\left.\frac{\Gamma(1+2 \alpha)}{\Gamma(1+\alpha)} \cdot \frac{\Gamma(1+\alpha) t^{2 \alpha}}{\Gamma(1+2 \alpha)}\right|_{0} ^{1}-1^{\alpha}\right) \\
& \times \frac{1}{\Gamma(1+\alpha)} \int_{0}^{1} f(u)(\mathrm{d} u)^{\alpha} \\
= & \left(\left.t^{2 \alpha}\right|_{0} ^{1}-1^{\alpha}\right) \frac{1}{\Gamma(1+\alpha)} \int_{0}^{1} f(u)(\mathrm{d} u)^{\alpha}=0 .
\end{aligned}
$$


Moreover, we have

$$
\begin{aligned}
& H^{(\alpha)}(0)=f^{*}(0)-f(0)=f^{*}(0)=0 \\
& H(x)= f^{*}(x)-f(x) \\
&= \frac{\Gamma(1+2 \alpha) x^{\alpha}}{\Gamma(1+\alpha)} \\
& \cdot \frac{1}{\Gamma(1+\alpha)} \int_{0}^{1} f(u)(\mathrm{d} u)^{\alpha}-f(x) \\
&= \frac{\Gamma(1+2 \alpha) x^{\alpha}}{\Gamma(1+\alpha)} \\
& \times\left(\frac{1}{\Gamma(1+\alpha)} \int_{0}^{1} f(u)(\mathrm{d} u)^{\alpha}\right. \\
&\left.\quad-\frac{f(x)}{\Gamma(1+2 \alpha) x^{\alpha} / \Gamma(1+\alpha)}\right) \quad(x \in(0,1]) .
\end{aligned}
$$

Since the function

$$
\frac{f(x)}{\Gamma(1+2 \alpha) x^{\alpha} / \Gamma(1+\alpha)}
$$

is increasing on $(0,1]$, we can see that

$$
\frac{H^{(\alpha)}(x)}{\Gamma(1+2 \alpha) x^{\alpha} / \Gamma(1+\alpha)}
$$

is decreasing on $(0,1]$.

Next, we show that $H(x) \geqq 0$ on $[0,1]$. This proof can be divided into the following two parts.

(a) Assume that there exists a point $x_{0} \in(0,1)$ such that

$$
\frac{H^{(\alpha)}\left(x_{0}\right)}{\Gamma(1+2 \alpha) x_{0}^{\alpha} / \Gamma(1+\alpha)}=0 \text {. }
$$

Then

$$
\begin{aligned}
& H^{(\alpha)}(x) \geqq 0 \quad\left(x \in\left[0, x_{0}\right]\right), \\
& H^{(\alpha)}(x) \leqq 0 \quad\left(x \in\left[x_{0}, 1\right]\right) .
\end{aligned}
$$

Hence we assume that $x \in\left[0, x_{0}\right]$. Then $H(x) \geqq H(0)=0$. If we assume that $x \in\left[x_{0}, 1\right]$, then $H(x) \geqq H(1)=0$ on $\left[x_{0}, 1\right]$. Thus $H(x) \geqq 0$ on $[0,1]$.

(b) Suppose that

$$
\frac{H^{(\alpha)}(x)}{\Gamma(1+2 \alpha) x^{\alpha} / \Gamma(1+\alpha)}>0 \quad(x \in(0,1)) .
$$

In this case, $H(x)$ is increasing on $[0,1)$. Hence $H(x) \geqq$ $H(0)=0$ on $[0,1)$. It follows from $H(1)=0$ that $H(x) \geqq 0$ on $[0,1]$. Thus, by applying a known result [5, Theorem 2.28], we have

$$
\begin{aligned}
& \frac{1}{\Gamma(1+\alpha)} \int_{0}^{1}\left[f(x)-f^{*}(x)\right] g(x)(\mathrm{d} x)^{\alpha} \\
& =-\frac{1}{\Gamma(1+\alpha)} \int_{0}^{1} H^{(\alpha)}(x) g(x)(\mathrm{d} x)^{\alpha} \\
& =-\left(\left.H^{(\alpha)}(x) g(x)\right|_{0} ^{1}\right. \\
& \left.\quad-\frac{1}{\Gamma(1+\alpha)} \int_{0}^{1} g^{(\alpha)}(x) H(x)(\mathrm{d} x)^{\alpha}\right) \\
& =\frac{1}{\Gamma(1+\alpha)} \int_{0}^{1} g^{(\alpha)}(x) H(x)(\mathrm{d} x)^{\alpha} \geqq 0,
\end{aligned}
$$

because $H(x) \geqq 0$ and $g(x)$ is increasing (and hence $g^{(\alpha)}(x) \geqq$ $0)$. We have thus completed our proof.

We are in a position to state and prove our main result as follows.

Theorem 15. Let $f_{1}(x), f_{2}(x), \ldots, f_{n}(x) \in C_{\alpha}(0,1)$ with $f_{i}(0)=0$ and

$$
\frac{f_{i}(x)}{\Gamma(1+2 \alpha) x^{\alpha} / \Gamma(1+\alpha)}
$$

increasing on $(0,1]$ for $i=1,2, \ldots, n$. If $f_{i}(x)(i=1,2, \ldots, n)$ is increasing on $(0,1]$, then

$$
\begin{aligned}
& \frac{1}{\Gamma(1+\alpha)} \int_{0}^{1} \prod_{i=1}^{n} f_{i}(x)(\mathrm{d} x)^{\alpha} \\
& \geqq\left(\frac{[\Gamma(1+2 \alpha)]^{n}}{[\Gamma(1+\alpha)]^{n}} \frac{1}{\Gamma(1+\alpha)} \int_{0}^{1} x^{n \alpha}(\mathrm{d} x)^{\alpha}\right) \\
& \quad \times\left(\prod_{i=1}^{n} \frac{1}{\Gamma(1+\alpha)} \int_{0}^{1} f_{i}(u)(\mathrm{d} u)^{\alpha}\right) .
\end{aligned}
$$

Proof. It follows from Lemma 14 and the increasing property of $f_{i}(x)(i=1,2, \ldots, n)$ that, for $f_{i}^{*}(x)$ defined as in Lemma 14

$$
\begin{aligned}
& \frac{1}{\Gamma(1+\alpha)} \int_{0}^{1} \prod_{i=1}^{n} f_{i}(x)(\mathrm{d} x)^{\alpha} \\
& =\frac{1}{\Gamma(1+\alpha)} \int_{0}^{1} f_{n}(x) \prod_{i=1}^{n-1} f_{i}(x)(\mathrm{d} x)^{\alpha} \\
& \geqq \frac{1}{\Gamma(1+\alpha)} \int_{0}^{1} f_{n}^{*}(x) \prod_{i=1}^{n-1} f_{i}(x)(\mathrm{d} x)^{\alpha}
\end{aligned}
$$




$$
\begin{aligned}
& =\frac{1}{\Gamma(1+\alpha)} \\
& \times \int_{0}^{1}\left(\frac{\Gamma(1+2 \alpha) x^{\alpha}}{\Gamma(1+\alpha)} \cdot \frac{1}{\Gamma(1+\alpha)} \int_{0}^{1} f_{n}(u)(\mathrm{d} u)^{\alpha}\right) \\
& \times \prod_{i=1}^{n-1} f_{i}(x)(\mathrm{d} x)^{\alpha} \\
& =\frac{1}{\Gamma(1+\alpha)} \int_{0}^{1} \frac{\Gamma(1+2 \alpha) x^{\alpha}}{\Gamma(1+\alpha)} \prod_{i=1}^{n-1} f_{i}(x)(\mathrm{d} x)^{\alpha} \\
& =\frac{1}{\Gamma(1+\alpha)} \int_{0}^{1} f_{n}(u)(\mathrm{d} u)^{\alpha} \\
& \times \frac{1}{\Gamma(1+\alpha)} \int_{0}^{1} f_{n-1}(x) \frac{\Gamma(1+2 \alpha) x^{\alpha}}{\Gamma(1+\alpha)} \prod_{i=1}^{n-2} f_{i}(x)(\mathrm{d} x)^{\alpha} \\
& \geqq \frac{1}{\Gamma(1+\alpha)} \int_{0}^{1} f_{n}(u)(\mathrm{d} u)^{\alpha} \\
& \times \frac{1}{\Gamma(1+\alpha)} \int_{0}^{1} f_{n-1}^{*}(x) \frac{\Gamma(1+2 \alpha) x^{\alpha}}{\Gamma(1+\alpha)} \prod_{i=1}^{n-2} f_{i}(x)(\mathrm{d} x)^{\alpha} \\
& =\frac{1}{\Gamma(1+\alpha)} \int_{0}^{1} f_{n}(u)(\mathrm{d} u)^{\alpha} \\
& \times \frac{1}{\Gamma(1+\alpha)} \int_{0}^{1} f_{n-1}(u)(\mathrm{d} u)^{\alpha} \\
& \frac{1}{\Gamma(1+\alpha)} \int_{0}^{1} \frac{[\Gamma(1+2 \alpha)]^{2} x^{2 \alpha}}{[\Gamma(1+\alpha)]^{2}} \prod_{i=1}^{n-2} f_{i}(x)(\mathrm{d} x)^{\alpha} \\
& =\frac{1}{\Gamma(1+\alpha)} \int_{0}^{1} f_{n}(u)(\mathrm{d} u)^{\alpha} \\
& \times \frac{1}{\Gamma(1+\alpha)} \int_{0}^{1} f_{n-1}(u)(\mathrm{d} u)^{\alpha} \\
& \cdot \frac{1}{\Gamma(1+\alpha)} \int_{0}^{1} \frac{[\Gamma(1+2 \alpha)]^{2} x^{2 \alpha}}{[\Gamma(1+\alpha)]^{2}} f_{n-2}(x) \prod_{i=1}^{n-3} f_{i}(x)(\mathrm{d} x)^{\alpha} \\
& \geqq \frac{1}{\Gamma(1+\alpha)} \int_{0}^{1} f_{n}(u)(\mathrm{d} u)^{\alpha} \\
& \times \frac{1}{\Gamma(1+\alpha)} \int_{0}^{1} f_{n-1}(u)(\mathrm{d} u)^{\alpha} \\
& \times \frac{1}{\Gamma(1+\alpha)} \int_{0}^{1} f_{n-2}(u)(\mathrm{d} u)^{\alpha} \\
& \cdot \frac{1}{\Gamma(1+\alpha)} \int_{0}^{1} \frac{[\Gamma(1+2 \alpha)]^{3} x^{3 \alpha}}{[\Gamma(1+\alpha)]^{3}} f_{n-2}(x) \prod_{i=1}^{n-3} f_{i}(x)(\mathrm{d} x)^{\alpha} \\
& \geqq \cdots \geqq \cdots \\
& =\prod_{i=1}^{n} \frac{1}{\Gamma(1+\alpha)} \int_{0}^{1} f_{i}(u)(\mathrm{d} u)^{\alpha}
\end{aligned}
$$

which yields

$$
\begin{aligned}
& \frac{1}{\Gamma(1+\alpha)} \int_{0}^{1} \prod_{i=1}^{n} f_{i}(x)(\mathrm{d} x)^{\alpha} \\
& \geq\left(\frac{[\Gamma(1+2 \alpha)]^{n}}{[\Gamma(1+\alpha)]^{n}} \frac{1}{\Gamma(1+\alpha)} \int_{0}^{1} x^{n \alpha}(\mathrm{d} x)^{\alpha}\right) \\
& \quad \times\left(\prod_{i=1}^{n} \frac{1}{\Gamma(1+\alpha)} \int_{0}^{1} f_{i}(u)(\mathrm{d} u)^{\alpha}\right) .
\end{aligned}
$$

The proof of Theorem 15 is thus completed.

Remark 16. In its special case when $\alpha=1$, the inequality (36) asserted by Theorem 15 would reduce to the Anderson-Fink inequality (2).

\section{Conflict of Interests}

The authors declare that they have no conflict of interests regarding the publication of this paper.

\section{Acknowledgments}

This work was supported by the Scientific Research Program Funded by Shaanxi Provincial Education Department (no. 2013JK1139), the China Postdoctoral Science Foundation (no. 2013M542370), and the Specialized Research Fund for the Doctoral Program of Higher Education of China (Grant no. 20136118120010). This study was also supported by the National Natural Science Foundation of China (nos. 11301414, 11226173, and 61272283).

\section{References}

[1] B. Z. Anderson, "An inequality for convex functions," Nordisk Matematisk Tidsskrift, vol. 6, pp. 25-26, 1958.

[2] A. M. Fink, “Andersson's inequality," Mathematical Inequalities and Applications, vol. 6, no. 2, pp. 241-245, 2003.

[3] X.-J. Yang, Local Fractional Functional Analysis and Its Applications, Asian Academic, Hong Kong, 2011.

[4] X.-J. Yang, "Local fractional integral transforms," Progress in Nonlinear Science, vol. 4, pp. 1-225, 2011.

[5] X.-J. Yang, Advanced Local Fractional Calculus and Its Applications, World Science, New York, NY, USA, 2012.

[6] W.-H. Su, D. Baleanu, X.-J. Yang, and H. Jafari, "Damped wave equation and dissipative wave equation in fractal strings within the local fractional variational iteration method," Fixed Point Theory and Applications, vol. 2013, no. 1, article 89, 2013. 
[7] M.-S. Hu, D. Baleanu, and X.-J. Yang, "One-phase problems for discontinuous heat transfer in fractal media," Mathematical Problems in Engineering, vol. 2013, Article ID 358473, 3 pages, 2013.

[8] K. M. Kolwankar and A. D. Gangal, "Hölder exponents of irregular signals and local fractional derivatives," PramanaJournal of Physics, vol. 48, no. 1, pp. 49-68, 1997.

[9] K. M. Kolwankar and A. D. Gangal, "Local fractional FokkerPlanck equation,” Physical Review Letters, vol. 80, no. 2, pp. 214217, 1998.

[10] G. Jumarie, "The Minkowski's space-time is consistent with differential geometry of fractional order," Physics Letters A, vol. 363, no. 1-2, pp. 5-11, 2007.

[11] G. Jumarie, "Modified Riemann-Liouville derivative and fractional Taylor series of nondifferentiable functions further results," Computers and Mathematics with Applications, vol. 51, no. 9-10, pp. 1367-1376, 2006.

[12] A. Parvate and A. D. Gangal, "Calculus on fractal subsets of real line. I: formulation," Fractals, vol. 17, no. 1, pp. 53-81, 2009.

[13] A. Parvate and A. D. Gangal, "Fractal differential equations and fractal-time dynamical systems," Pramana-Journal of Physics, vol. 64, no. 3, pp. 389-409, 2005.

[14] W. Chen, "Time-space fabric underlying anomalous diffusion," Chaos, Solitons and Fractals, vol. 28, no. 4, pp. 923-929, 2006.

[15] W. Chen, X.-D. Zhang, and D. Korošak, "Investigation on fractional and fractal derivative relaxation-oscillation models," International Journal of Nonlinear Sciences and Numerical Simulation, vol. 11, no. 1, pp. 3-9, 2010.

[16] W. Chen, H. Sun, X.-D. Zhang, and D. Korošak, "Anomalous diffusion modeling by fractal and fractional derivatives," Computers and Mathematics with Applications, vol. 59, no. 5, pp. 1754-1758, 2010.

[17] F. B. Adda and J. Cresson, "About non-differentiable functions," Journal of Mathematical Analysis and Applications, vol. 263, no. 2, pp. 721-737, 2001.

[18] J.-H. He, "A new fractal derivation," Thermal Science, vol. 15, no. 1, pp. S145-S147, 2011.

[19] J.-H. He, "Asymptotic methods for solitary solutions and compactons," Abstract and Applied Analysis, vol. 2012, Article ID 916793, 130 pages, 2012.

[20] J. Fan and J.-H. He, "Fractal derivative model for air permeability in hierarchic porous media," Abstract and Applied Analysis, vol. 2012, Article ID 354701, 7 pages, 2012.

[21] Y.-J. Yang, D. Baleanu, and X.-J. Yang, "A local fractional variational iteration method for Laplace equation within local fractional operators," Abstract and Applied Analysis, vol. 2013, Article ID 202650, 6 pages, 2013.

[22] F. Gao, X.-J. Yang, and Z. Kang, "Local fractional Newton's method derived from modified local fractional calculus," in Proceedings of the 2nd Scientifc and Engineering Computing Symposium on Computational Sciences and Optimization (CSO '09), pp. 228-232, IEEE Computer Society, Sanya, China, April 2009.

[23] X.-J. Yang and F. Gao, "The fundamentals of local fractional derivative of the one-variable nondifferentiable functions," World Sci-Tech R and D, vol. 31, no. 5, pp. 920-921, 2009.

[24] X.-J. Yang and F. Gao, "Fundamentals of local fractional iteration of the continuously nondifferentiable functions derived from local fractional calculus," in Proceedings of the International Conference on Computer Science and Information Engineering (CSIE '11), pp. 398-404, Springer, Zhengzhou, China, May 2011.
[25] X.-J. Yang, L. Li, and R. Yang, "Problems of local fractional definite integral of the one-variable nondifferentiable function," World Sci-Tech R and D, vol. 31, pp. 722-724, 2009.

[26] W.-H. Su, X.-J. Yang, H. Jafari, and D. Baleanu, "Fractional complex transform method for wave equations on Cantor sets within local fractional differential operator," Advances in Difference Equations, vol. 2013, article 97, 2013.

[27] G.-S. Chen, “The local fractional Stieltjes Transform in fractal space," Advances in Intelligent Transportation Systems, vol. 1, no. 1, pp. 29-31, 2012.

[28] G.-S. Chen, "Local fractional improper integral in fractal space," Advances in Information Technology and Management, vol. 1, no. 1, pp. 4-8, 2012.

[29] X.-J. Yang, D. Baleanu, H. M. Srivastava, and J. A. T. Machado, "On local fractional continuous wavelet transform," Abstract and Applied Analysis, vol. 2013, Article ID 725416, 5 pages, 2013.

[30] G.-S. Chen, "Mean value theorems for local fractional integrals on fractal space," Advances in Mechanical Engineering and Its Applications, vol. 1, no. 1, pp. 5-8, 2012.

[31] Y.-J. Yang, D. Baleanu, and X.-J. Yang, "Analysis of fractal wave equations by local fractional Fourier series method," Advances in Mathematical Physics, vol. 2013, Article ID 632309, 6 pages, 2013.

[32] G.-S. Chen, "The finite Yang-Laplace Transform in fractal space," Journal of Computational Science \& Engineering, vol. 6, pp. 363-365, 2013.

[33] Y. Zhao, D.-F. Cheng, and X.-J. Yang, "Approximation solutions for local fractional Schrödinger equation in the onedimensional Cantorian system," Advances in Mathematical Physics, vol. 2013, Article ID 291386, 5 pages, 2013.

[34] G.-S. Chen, "The local fractional Hilbert transform in fractal space," Journal of Applied Sciences and Engineering Innovation, vol. 1, pp. 21-27, 2014.

[35] D. Baleanu, J. A. T. Machado, C. Cattani, M. C. Baleanu, and X.-J. Yang, "Local fractional variational iteration and decomposition methods for wave equation on Cantor sets within local fractional operators," Abstract and Applied Analysis, vol. 2014, Article ID 535048, 6 pages, 2014.

[36] A. A. Kilbas, H. M. Srivastava, and J. J. Trujillo, Theory and Applications of Fractional Differential Equations, vol. 204 of North-Holland Mathematical Studies, Elsevier Science, NorthHolland, Amsterdam, The Netherlands, 2006.

[37] H. M. Srivastava, "Operators of fractional calculus and associated Mittag-Leffler type functions," in Proceedings of the 6th International Conference on Analytic Methods of Analysis and Differential Equations (AMADE '12), S. V. Rogosin, Ed., pp. 207-228, Publishing Center of the Belarusian State University, Minsk, Belarus, September 2012.

[38] X.-J. Ma, H. M. Srivastava, D. Baleanu, and X.-J. Yang, "A new Neumann series method for solving a family of local fractional fredholm and volterra integral equations," Mathematical Problems in Engineering, vol. 2013, Article ID 325121, 6 pages, 2013.

[39] Y.-J. Hao, H. M. Srivastava, H. Jafari, and X.-J. Yang, "Helmholtz and diffusion equations associated with local fractional derivative operators involving the Cantorian and Cantor-type cylindrical coordinates," Advances in Mathematical Physics, vol. 2013, Article ID 754248, 5 pages, 2013.

[40] X.-J. Yang, H. M. Srivastava, J.-H. He, and D. Baleanu, "Cantortype cylindrical-coordinate method for differential equations with local fractional derivatives," Physics Letters A, vol. 377, no. 28-30, pp. 1696-1700, 2013. 
[41] A.-M. Yang, Z.-S. Chen, H. M. Srivastava, and X.-J. Yang, "Application of the local fractional series method and the variational iteration method to the Helmhotz equation involving local fractional derivative operators," Abstract and Applied Analysis, vol. 2013, Article ID 259125, 6 pages, 2013.

[42] H. Mo, X. Sui, and D. Yu, "Generalized convex functions andsome inequalities on fractal sets," submitted, http://arxiv.org/ abs/1404.3964. 


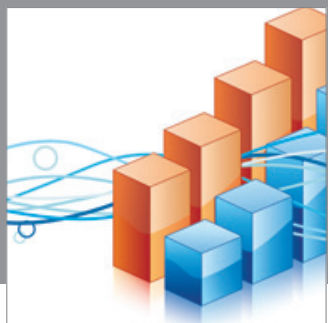

Advances in

Operations Research

mansans

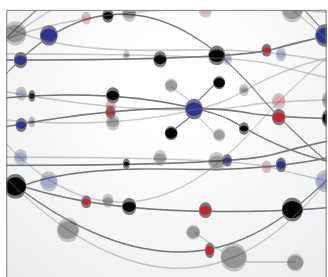

The Scientific World Journal
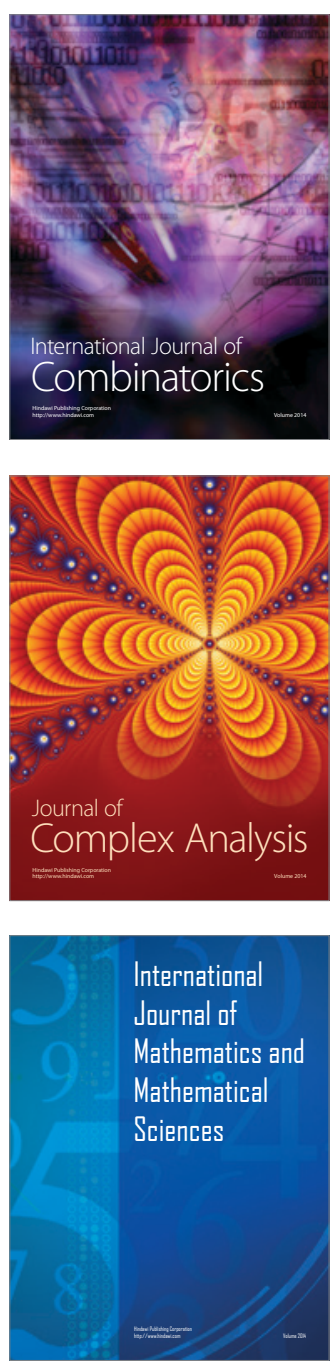
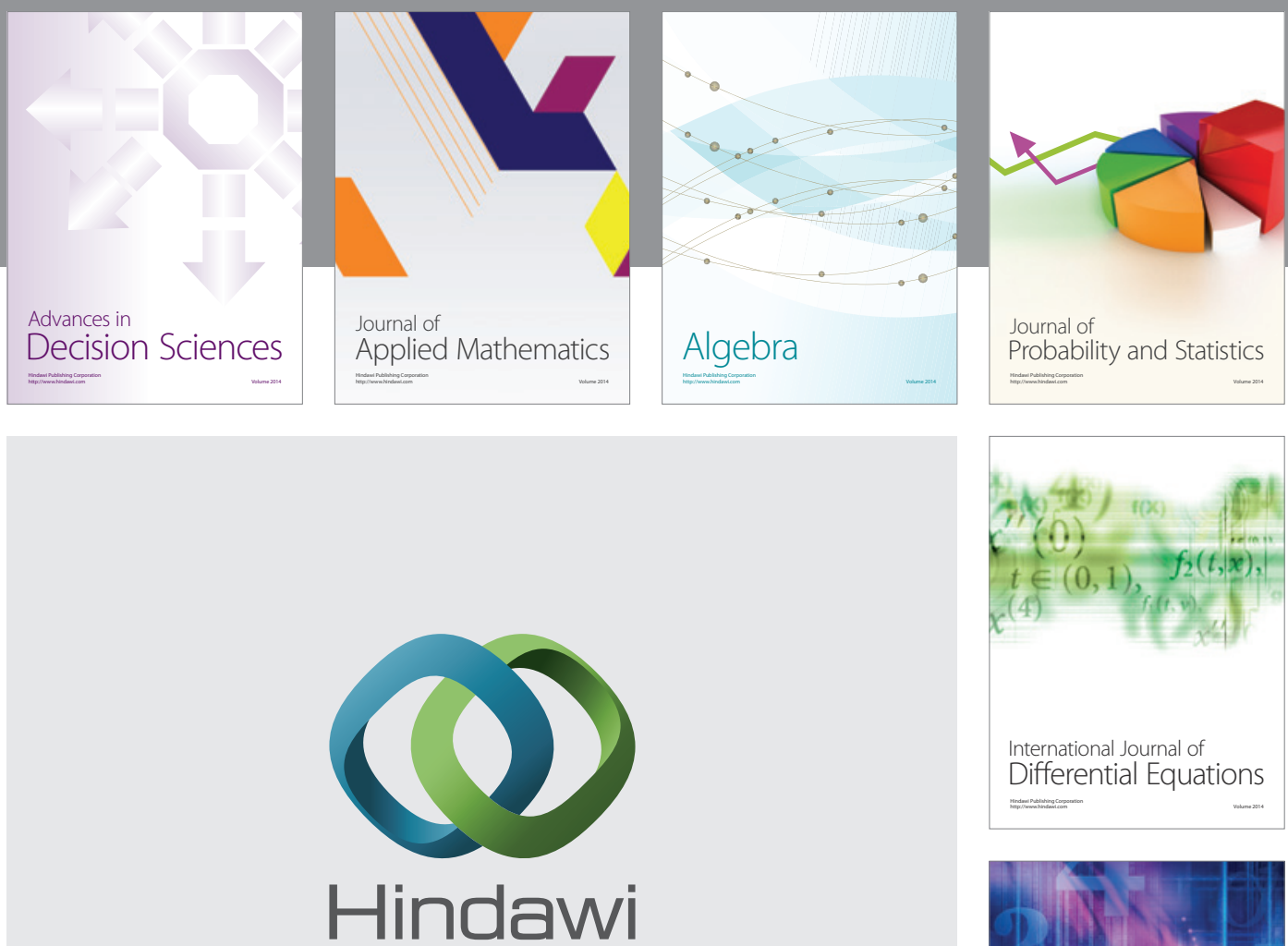

Submit your manuscripts at http://www.hindawi.com
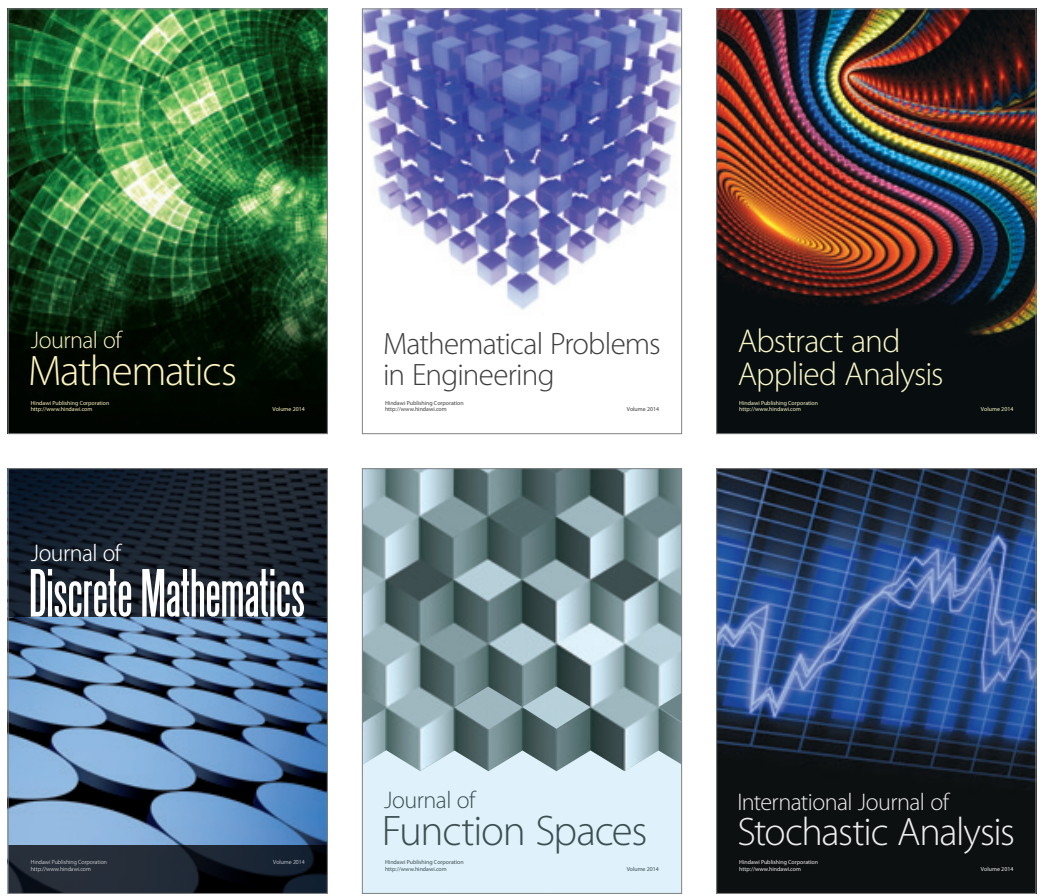

Journal of

Function Spaces

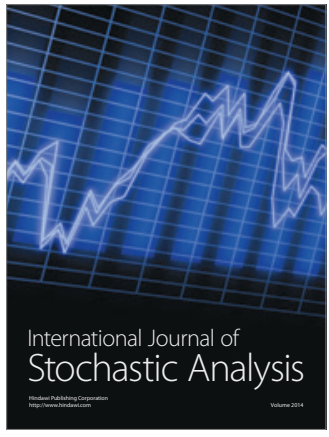

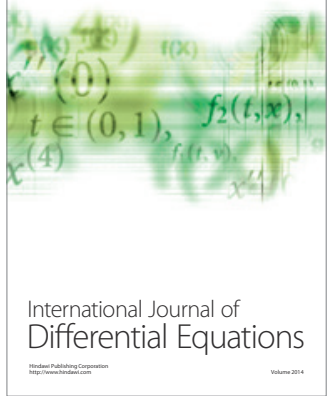
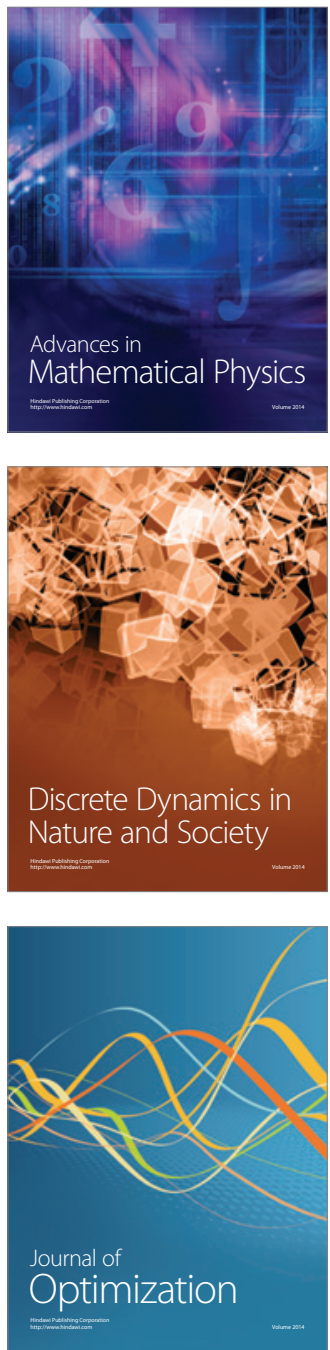